\title{
Constructions, reconstructions et commémorations clunisiennes, 1790-2010
}

Cluny, 13-15 mai 2010

\section{Didier Méhu}

\section{(2) OpenEdition}

\section{Journals}

\section{Édition électronique}

URL : https://journals.openedition.org/cem/11953

DOI : $10.4000 /$ cem. 11953

ISSN : 1954-3093

Éditeur

Centre d'études médiévales Saint-Germain d'Auxerre

Édition imprimée

Pagination : 131-137

ISSN : $1623-5770$

\section{Référence électronique}

Didier Méhu, «Constructions, reconstructions et commémorations clunisiennes, 1790-2010 », Bulletin du centre d'études médiévales d'Auxerre | BUCEMA [En ligne], 15 | 2011, mis en ligne le 23 septembre 2011, consulté le 22 septembre 2022. URL : http://journals.openedition.org/cem/11953 ; DOI : https:// doi.org/10.4000/cem.11953

Ce document a été généré automatiquement le 22 septembre 2022.

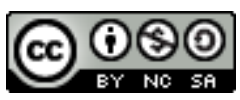

Creative Commons - Attribution - Pas d'Utilisation Commerciale - Partage dans les Mêmes Conditions 4.0 International - CC BY-NC-SA 4.0

https://creativecommons.org/licenses/by-nc-sa/4.0/ 


\title{
Constructions, reconstructions et commémorations clunisiennes, 1790-2010
}

Cluny, 13-15 mai 2010

\author{
Didier Méhu
}

1 Ce colloque s'est tenu dans le cadre des célébrations du $11^{\mathrm{e}}$ centenaire de l'abbaye de Cluny. Il s'agissait du premier des trois colloques co-organisés par le Centre d'études médiévales d'Auxerre, les deux autres, de nature plus classique, visaient à réfléchir aux relations entre le monachisme et la société occidentale au "premier âge féodal » ${ }^{1}$. Cette rencontre portait sur l'histoire récente de Cluny, depuis la dissolution de l'abbaye en 1790 jusqu'aux présentes cérémonies commémoratives. Le but était de réfléchir à la manière dont l'objet historique Cluny a été construit, reconstruit et transformé pendant ces deux siècles, et placer ainsi l'activité historienne dans le temps de la lente élaboration du discours scientifique moderne, de la conservation des Monuments historiques, du discours patrimonial, des pratiques sociales liées à la célébration collective de la mémoire. Il s'agissait également d'interroger la place des historiens dans les pratiques commémoratives, qu'ils en soient les initiateurs - colloques au moment d'un centenaire -, les participants actifs ou secondaires - en tant qu'experts ou conférenciers invités - ou qu'ils en soient exclus.

Le projet a été conçu dès le départ (soit en 2005) dans le souci de conjoindre la réflexion historienne à celle des "acteurs" de la commémoration et de la reconstruction clunisienne contemporaine, à savoir les services de conservation et de restauration des Monuments historiques, les associations touristiques et "culturelles", les ingénieurs de l'École nationale des arts et métiers, dont le directeur est devenu maire de Cluny en 2008. La rencontre se voulait aussi largement ouverte au public clunisois - au sens large, soit les habitants de Cluny et des environs, voire ceux des "sites clunisiens " réunis de manière informelle au sein de la Fédération des sites clunisiens depuis 1991 et largement sollicités tout au long des cérémonies commémoratives de 2009-2010. La 
publicité du colloque a été faite à l'intention de ces différents acteurs, que nous souhaitions voir nombreux et impliqués dans nos discussions. In fine, une partie de ces objectifs n'a pas été atteinte, tant parce que plusieurs « acteurs » de la commémoration ont annulé leur participation au colloque que parce que le public était peu nombreux et que la publicité de la rencontre a été très mal relayée sur le plan local.

3 Le colloque s'est déroulé sur quatre demi-journées, du jeudi 13 mai après-midi au samedi 15 mai au matin, au théâtre municipal de Cluny. Les communications étaient réunies par groupe de deux ou trois autour d'un même thème, puis suivies d'une période de discussions. Le programme avait été établi de telle sorte que chaque communicant dispose de 35 à 40 minutes pour exposer son propos et que le temps prévu pour les discussions permette à chacun de s'exprimer à sa guise.

4 La première session visait à poser les termes de la réflexion, soit la place des historiens dans le phénomène commémoratif, tant général que proprement clunisien.

5 Le maire de Cluny, M. Jean-Luc Delpeuch, a vivement introduit les débats en indiquant que la programmation coordonnée par la municipalité et la Fédération européenne des sites clunisiens correspondait davantage à des "festivités ", résolument tournées vers le futur et la rencontre, qu'à des « commémorations » nostalgiques. Il a émis l'idée que des sociologues ou des anthropologues auraient une légitimité plus forte que des historiens pour analyser des événements du temps présent, d'autant que les historiens sont eux-mêmes les acteurs de ce qu'ils se proposent d'étudier : le nombre important de colloques et conférences organisés par des historiens dans le cadre de « Cluny 2010 » en témoigne. Il a souligné le risque que les présupposés des chercheurs sur les « commémorations » et leur propension à s'ériger en gardiens d'une orthodoxie en la matière, expliquent leur difficulté à entrer en échange avec les autres acteurs de ces événements.

6 Didier Méhu - professeur d'histoire et d'histoire de l'art du Moyen Âge, université de Laval, Québec - a rappelé la démarche qui a présidé à l'organisation du colloque, soit celle de profiter de l'occasion, non pas pour commémorer Cluny, mais pour amorcer une réflexion globale, mêlant historiens et non-historiens, sur les pratiques commémoratives dans lesquelles nous sommes tous impliqués aujourd'hui et qui constituent des moments forts du calendrier social. Dans une telle perspective, la seule attitude raisonnable est de ne pas commémorer, ni même, pour les historiens, de tenir des rencontres scientifiques opportunistes au moment de la commémoration, mais d'inviter le corps social à déconstruire les pratiques mémorielles et commémoratives qui sont considérées comme allant de soi.

7 Dominique Iogna-Prat - historien du Moyen Âge, directeur de recherche au CNRS, Paris - a situé les célébrations actuelles dans l'histoire des commémorations clunisiennes depuis la fin du xix siècle, afin d'en dégager les enjeux non-conscients et les principales orientations sociales et politiques. Dans cette perspective, les commémorations de 2009-2010 se caractérisent par leur émiettement, l'absence de ligne directrice, la coexistence non-pensée du spirituel, du festif et du mémoriel et la dissolution de la réflexion des historiens professionnels dans un ensemble d'initiatives à coloration historique inscrites dans une démarche non institutionnelle et majoritairement privée.

8 La deuxième session a été consacrée à une mise en perspective de deux moments forts de la destruction-reconstruction de Cluny. 
9 Yann Potin - historien du Moyen Âge, chargé d'études documentaires aux Archives nationales, Paris - a rouvert, à la lumière de documents inédits, le dossier de la destruction de l'abbaye au début du $\mathrm{XIX}^{\mathrm{e}}$ siècle en la mettant en perspective avec le contexte révolutionnaire national et les attitudes des locaux. Il en ressort l'absence d'une attitude univoque, sachant que la volonté marquée de mettre un terme au monachisme clunisien et à ses privilèges ne s'est accompagnée d'aucun projet concret de destruction des bâtiments avant 1815, mais que ni les Clunisois ni l'État n'ont manifesté d'opposition réelle devant la destruction qui s'est opérée pendant la Restauration.

10 La situation de la fin du $\mathrm{XIX}^{\mathrm{e}}$ siècle présentée par Alain Rauwel - historien du Moyen Âge, professeur agrégé à l'université de Bourgogne - présente une certaine continuité, puisque la tentative de restauration d'une communauté monastique à Cluny sous l'égide de dom Mayeul Lamey, entre 1888 et 1896, qui s'inscrit pourtant dans une forte période de reconquête catholique en France, s'est avérée un échec par son absence de soutien, tant de la part des Clunisois que des autorités civiles ou ecclésiastiques.

11 La seconde journée du colloque a été consacrée aux modalités de la construction des discours scientifique, littéraire et fictionnel autour de l'abbaye de Cluny au cours des $\mathrm{XIX}^{\mathrm{e}}$ et $\mathrm{XX}^{\mathrm{e}}$ siècles.

12 Isabelle Vernus - historienne du Moyen Âge, directrice des archives départementales de Saône-et-Loire - est revenue sur le «trou noir » dans lequel les archives de l'abbaye ont été laissées pendant les deux premiers tiers du $\mathrm{XIX}^{\mathrm{e}}$ siècle, et ce, tant par le désintérêt total de la municipalité de l'époque à l'égard des archives que par le manque de mesures concrètes prises par les autorités de l'État pour en assurer la conservation adéquate. L'état actuel des fonds, éclatés entre plusieurs dépôts et pour lesquels aucun inventaire complet n'est disponible, et ce malgré la notoriété considérable acquise par les « chartes » de l'abbaye de Cluny dans le discours des médiévistes, en est le résultat déplorable. La récente acquisition par les archives départementales de Saône-et-Loire d'un fonds d'une douzaine de documents originaux des $\mathrm{XI}^{\mathrm{e}}-\mathrm{XV}^{\mathrm{e}}$ siècles, auparavant conservés dans une collection particulière, et inconnus de tous les chercheurs, montre l'énorme travail qu'il reste à accomplir pour conserver l'héritage culturel de Cluny.

13 S'inscrivant dans le sillage de cette réflexion, Alain Guerreau - historien du Moyen Âge, directeur de recherche au CNRS, Paris - a insisté sur l'obstacle majeur au progrès des connaissances produit par l'émiettement généralisé qui caractérise le discours scientifique actuel, qu'il concerne la conservation des documents ou les champs de la recherche historique. L'histoire de Cluny, monument s'il en est de l'historiographie médiévale, ne s'inscrit dans aucune perspective d'ensemble visant à permettre la compréhension de la logique globale de la société dans laquelle Cluny s'est développé et avait un sens. La situation actuelle, malgré d'innombrables titres publiés, est donc peu différente de celle dans laquelle on se trouvait au milieu du XIX ${ }^{\mathrm{e}}$ siècle, à l'époque où l'on commença de s'intéresser à l'histoire de Cluny. Le seul moyen de s'en sortir est de lutter contre les monopoles sectaires - micro-études indépendantes les unes des autres et fondées sur de putatives spécialités -, de construire des concepts appropriés aux structures spécifiques de la société médiévale susceptibles de saisir une logique globale, qui donne accès à des évolutions partielles que l'on observe à différentes échelles.

14 Un ensemble de cinq communications a ensuite porté sur la place occupée par Cluny dans la constitution progressive de l'histoire et de l'histoire de l'art du Moyen Âge. 
Laurent Baridon - professeur d'histoire de l'art contemporain, université de Grenoble 2 - et Didier Méhu se sont penchés sur l'œuvre des historiens français de la première moitié du XIx ${ }^{e}$ siècle - Augustin Thierry, François Guizot, Jules Michelet - et sur celle d'Eugène Viollet-le-Duc. Il en ressort une ignorance frappante de Cluny dans les histoires de France rédigées par les fondateurs de la discipline, et ce jusqu'à ce que Viollet-le-Duc fasse de l'abbaye bourguignonne - dans les années 1860 - le creuset du renouveau progressiste de la société médiévale, qu'il dépeint dans une dynamique antiféodale préfigurant la société bourgeoise et libérale de son temps. Parallèlement, Cluny trouve sa place dans des œuvres sans envergure produites par des historiens régionaux, fortement marqués par un catholicisme revanchard, qui voit en Cluny l'exemple emblématique du martyr de la société libérale.

Cette perspective fut prolongée par l'intervention de Nicolas Reveyron - professeur d'histoire de l'art du Moyen Âge, université Lyon 2, Institut universitaire de France - au sujet de François Cucherat (1812-1887), prêtre originaire du Brionnais et auteur d'un ouvrage sur l'influence religieuse, intellectuelle et politique de Cluny au $\mathrm{xI}^{\mathrm{e}}$ siècle, primé par l'académie de Mâcon et plusieurs fois réédité dans la seconde moitié du $\mathrm{XIX}^{\mathrm{e}}$ siècle. La réhabilitation de Cluny souhaitée par Cucherat s'inscrit dans un projet de reconquête de la société contemporaine par les idéaux catholiques et l'auteur, très attaché à l'abbé Hugues, dont il possédait des reliques, le montre, dans son œuvre, comme le modèle à suivre pour cette reconquête.

Retournant à l'histoire de l'art institutionnelle, Daniel Russo - professeur d'histoire de l'art du Moyen Âge, université de Bourgogne, Institut universitaire de France - a considéré la place de Cluny dans l'œuvre de deux pères de la discipline en France au début du xx ${ }^{e}$ siècle, Émile Mâle et Henri Focillon. Cluny acquiert chez Mâle un statut de personnage littéraire et historique. Comme la cathédrale de Chartres pour l'art gothique, Cluny est l'incarnation du génie français, de l'art roman qui s'épanouit en Bourgogne et c'est un moteur de l'histoire de l'art. Ses propos sont repris par Focillon, qui les inscrit dans une perspective plus large, celle de l'ordre de cluny et des monastères de pèlerinages, reprenant à son compte les hypothèses formulées dans les années 1920 par Arthur Kingsley Porter.

17 C'est sur ce dernier que s'est arrêtée Kathryn Brush - professeur d'histoire de l'art du Moyen Âge, London, Canada, University of Western Ontario. En se fondant sur l'étude des archives inédites de l'historien de l'art et sur son œuvre publiée, elle a montré la manière dont il a construit Cluny comme la véritable matrice de l'essor architectural et artistique de l'Europe occidentale à partir du $\mathrm{xl}^{\mathrm{e}}$ siècle. On voit ainsi, dans l'œuvre d'Émile Mâle, d'Arthur Kingsley Porter et d'Henri Focillon, que le désintérêt, qui frappait l'abbaye bourguignonne au cours du XIX ${ }^{e}$ siècle, a fait place à une focalisation sur la puissance créatrice de Cluny, dans une démarche qui doit beaucoup aux historiens catholiques. Cluny incarne alors le génie civilisateur de l'Europe occidentale face à la barbarie ambiante, position qui n'est pas sans rappeler celle qui structure l'essentiel des initiatives visant à commémorer, ou célébrer, «l'esprit de Cluny » en 2010.

18 Ce socle historiographique et idéologique a constitué le creuset sur lequel s'est développée l'œuvre de Kenneth John Conant, architecte et archéologue célèbre pour avoir sorti de l'ombre l'église abbatiale détruite au début du xix ${ }^{e}$ siècle. Prolongeant les conclusions auxquelles elle avait abouti dans un ouvrage publié en 2007, Janet Marquardt - professeur d'histoire de l'art du Moyen Âge, Charleston, Eastern Illinois 
University - a montré la manière dont le Cluny de Conant était en grande partie le produit d'une représentation idéalisée de l'Église médiévale, incarnée dans un édifice aux proportions harmonieuses, idéales et gigantesques, représentatives de l'esprit triomphant $\mathrm{du} \mathrm{xII}^{\mathrm{e}}$ siècle, de la même manière que le transatlantique Queen Mary pouvait l'être de la société industrielle américaine des années 1930. Si Conant a entrepris des fouilles sur le site de l'ancienne abbatiale, les reconstructions qu'il a données de l'église et des bâtiments conventuels sont en grande partie le fruit de sa propre imagination créatrice, celle d'un architecte idéaliste, fasciné par la grandeur de l'Église médiévale.

19 Les deux dernières communications de la journée ont franchi le pas de l'imaginaire pour examiner la manière dont Cluny avait alimenté la littérature et la fiction cinématographique. Élizabeth Emery - professeur de lettres modernes, Montclair State University, New Jersey - s'est penchée sur la place de Cluny dans la littérature française $\mathrm{du} \mathrm{XIX}^{\mathrm{e}}$ siècle. Il est frappant de constater des parallèles importants avec l'histoire et l'histoire de l'art. Si l'on ne trouve que de rares allusions à l'abbaye bourguignonne dans la littérature antérieure à 1880 - mis à part chez Alphonse de Lamartine, implanté dans la région -, force est de reconnaître une recrudescence à la fin du siècle, que l'on doit majoritairement à Huysmans. D'À rebours à L'oblat, Cluny est de plus en plus présent, à mesure que le cheminement personnel de l'auteur et de son héros s'oriente vers le cloître. Cluny est l'idéal de la pureté, du labeur et de la conversion, celui qui fait triompher l'art et la poésie sur la vulgarité de la vie mondaine, autrement dit un modèle dans lequel le siècle décadent se devrait de puiser...

La fiction cinématographique, abordée par François Amy de la Bretèque - professeur d'histoire du cinéma, université de Montpellier 3 -, ne présente pas cette même vision, sans doute parce qu'elle ne s'inscrit pas dans un projet intellectuel moralisateur et parce que la spiritualité monastique n'est, pendant fort longtemps - ce qui n'est plus le cas aujourd'hui !-, très peu associée à l'évasion attendue du genre cinématographique. La fiction médiévale est chevaleresque, épique et diabolique, fort peu monastique. Lorsqu'elle le devient c'est, récemment, pour servir de cadre à des intrigues policières Le nom de la rose - ou dans des films confidentiels, ardus, fortement ancrés dans un souci de reconstitution historique et à forte teneur émotionnelle - Le voyage étranger de Serge Roulet, 1991. Et dans ces différentes œuvres, Cluny est absent.

La dernière demi-journée du colloque a été consacrée à la discussion autour de projets de reconstruction ou de reconstitution ayant lieu actuellement à Cluny.

Frédéric Didier - architecte en chef des Monuments historiques, responsable du site de l'abbaye de Cluny - a présenté le schéma directeur d'aménagement du site en insistant sur la nécessité de reconquérir la mémoire disparue et d'effacer partiellement les dommages causés par la destruction dramatique de l'église abbatiale. Partant du constat d'un échec général des politiques d'aménagement des sites abbatial et urbain depuis le $\mathrm{xIX}^{\mathrm{e}}$ siècle, il souhaite redonner une cohérence et une lisibilité aux articulations étroites entre ce double espace, qui est le fruit des 900 ans d'existence de l'abbaye. Le projet, entamé depuis les années 1990, articule des travaux de restauration des parties subsistantes des bâtiments monastiques, des reconstructions partielles permettant de mieux visualiser des sections fortement mutilées, la matérialisation de traces pour suivre l'ancienne emprise de l'abbatiale et des installations de "réalité augmentée ». Ces dernières, mises au point par les ingénieurs de l'École nationale supérieure des arts et métiers (ENSAM), aident à percevoir les dimensions et les formes 
de l'édifice disparu au moyen de bornes géo-référencées réparties sur le site. Les bornes transmettent des images numériques du bâtiment en intégrant les constructions encore en place, qu'elles prolongent virtuellement.

Ce projet de "réalité augmentée » a été présenté en détail par Guillaume Schotté ingénieur de l'ENSAM, Cluny -, en insistant sur la collaboration étroite entre les ingénieurs et les archéologues qui ont étudié le site ces dernières années. Dans le prolongement, Jean-François Coulais - géographe et ingénieur de recherche, ENSAM, Cluny - a présenté un projet en cours d'élaboration, qui vise à restituer de manière virtuelle - par le biais de la "réalité augmentée » - la continuité entre l'abbaye et son environnement urbain. Il s'agira, notamment, de compiler l'ensemble de la documentation archéologique et historique recueillie lors des dernières années sur le site de la ville - du Moyen Âge à l'époque moderne - en vue de proposer des reconstitutions virtuelles à différentes époques. Ces reconstitutions pourront déboucher sur des installations in situ, permettant de visualiser l'épaisseur historique du paysage urbain de Cluny.

Le colloque s'est achevé par une table ronde présidée par Anita Guerreau-Jalabert historienne du Moyen Âge, directrice de recherche au CNRS, Paris - à laquelle tous les communicants étaient conviés. La discussion a porté principalement sur la place des historiens dans les pratiques commémoratives, leurs réactions face à la «demande sociale » en matière d'histoire et de commémoration et les relations entre les historiens et les acteurs de la commémoration - agents de l'exécutif, associations, particuliers, etc. La parole a été donnée aux auditeurs pour apprécier, notamment, les impacts de la réflexion historienne à laquelle ils avaient assisté pendant ces deux jours. Outre les réactions reçues à chaud de manière orale, quelques remarques ont été formulées par écrit après le colloque.

Les textes présentés seront publiés dans un volume collectif, dont la parution aux Presses universitaires de Rennes est prévue en 2012. À ces textes seront ajoutées des réflexions de personnes n'ayant pas pu participer au colloque, notamment le maire de Cluny et les responsables de la Fédération des sites clunisiens, ainsi que les réactions formulées par écrit par certaines personnes qui ont assisté au colloque.

\section{NOTES}

1. Romainmôtier, du 24 au 26 juin 2010 ; Cluny, du 9 au 11 septembre 2010. Les comptes rendus figurent dans le présent volume. 
INDEX

Index géographique : France/Cluny

Mots-clés : colloque 